\title{
Cardiovascular System Findings Planned Time Point Number
}

National Cancer Institute

\section{Source}

National Cancer Institute. Cardiovascular System Findings Planned Time Point Number. NCI Thesaurus. Code C124031.

The numerical identifier of a cardiovascular system finding point in time. 